Issued by Sandia National Laboratories, operated for the United States Jepartment of Energy by Sandia Corporation.

NOTICE: This report was prepared as an account of work sponsored by an agency of the United States Government. Neither the United States Government, nor any agency thereof, nor any of their employees, nor any of their contractors, subcontractors, or their employees, make any warranty, express or implied, or assume any legal liability or responsibility for the accuracy, completeness, or usefulness of any information, apparatus, product, or process disclosed, or represent that its use would not infringe privately owned rights. Reference herein to any specific commercial product, process, or service by trade name, trademark, manufacturer, or otherwise, does not necessarily constitute or imply its endorsement, recommendation, or favoring by the United States Government, any agency thereof, or any of their contractors or subcontractors. The views and opinions expressed herein do not necessarily state or reflect those of the United States Government, any agency thereof, or any of their contractors.

Printed in the United States of America. This report has been reproduced directly from the best available copy.

Available to DOE and DOE contractors from

Office of Scientific and Technical Information

P.O. Box 62

Oak Ridge, TN 37831

Prices available from (703) 605-6000

Web site: http://www.ntis.gov/ordering.htm

Available to the public from

National Technical Information Service

U.S. Department of Commerce

5285 Port Royal Rd

Springfield, VA 22161

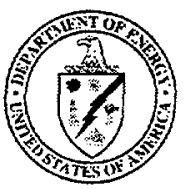




\section{DISCLAIMER}

Portions of this document may be illegible in electronic image products. Images are produced from the best available original document. 
SAND 2000-0141

Unlimited Release

Printed January 2000

\title{
LDRD Final Report on Intelligent Polymers for Nanodevice Performance Control
}

\author{
Gregory M. Jamison, David R. Wheeler and Randall S. Saunders \\ Organic Materials Department \\ Douglas A. Loy and Raafat Shaltout \\ Catalysis and Chemical Technologies Department \\ John A. Shelnutt \\ Biomolecular Materials and Interfaces Department \\ Martin J. Carr \\ Physical Sciences Department \\ Sandia National Laboratories \\ P. O. Box 5800 \\ Albuquerque, NM 87185-1407
}

\begin{abstract}
A variety of organic and hybrid organic-inorganic polymer systems were prepared and evaluated for their bulk response to optical, thermal and chemical environmental changes. These included modeling studies of polyene-bridged metal porphyrin sytems, metalmediated oligomerization of phosphaalkynes as heteroatomic analogues to polyacetylene monomers, investigations of chemically amplified degradation of acid- and base-sensitive polymers and thermally responsive thermoplastic thermosets based on Diels-Alder cycloaddition chemistry. The latter class of materials was utilized to initiate work to develop a new technique for rapidly building a library of systems with varying depolymerization temperatures.
\end{abstract}


CONTENTS

\section{PAGE}

I. Introduction..............................................

II. Results................................................. 8

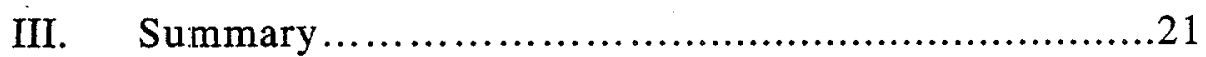




\section{LDRD Final Report on Intelligent Polymers for Nanodevice Performance Control}

\section{Introduction}

This project was designed to develop new classes of materials engineered to chemically respond to light, heat or chemical stimuli in a predictable manner. Materials characteristics which could be altered include optical, electrical or rheological properties. Potential applications for autonomous response materials might include sensors, actuators, encapsulation technologies, etc.

Research in smart materials has assumed a high priority in both industrial and government funded research programs over the past decade, as emerging technologies and markets develop. In general, smart materials are those in which the response to an external stimulus is part of a material's intrinsic properties; as such, harnessing the response of a material to stimuli has become the focus of smart materials development and integration in microactuators, sensors, micropumps, and multiple other applications. One of the most widely recognized smart materials systems is ceramic piezoelectric and electrostrictive materials, those which display a dimensional response to an electric field. Other types of intrinsically smart materials include magnetostrictive, electrochromic and electrorheological materials, and shape memory alloys (typically nickel alloys).

Less advanced are polymeric smart materials and systems in which they are integrated. Smart polymeric materials are attractive from weight, performance and ease-offabrication perspectives relative to inorganic materials, and platforms for their integration, such as MEMS, constitute a rapidly growing application area for electrostrictive polymers (often called artificial muscles) in particular. Polymeric smart materials fields include redox-switchable molecular wires composed of intrinsically conducting polymers and dimensionally dynamic gels (i.e. acrylamide copolymer systems for controlled drug release).

We have undertaken molecular modeling and experimental studies for the design and generation of novel autonomous response materials. These efforts include investigations of polyene-linked metal porphyrin complexes, to determine the degree of electrical communication as a function of polyene length, metal oxidation state and porphyrin conformation. Another investigation concerns the oligomerization behavior of phosphaalkynes, as heteroatomic acetylene analogues, under typical alkyne polymerization conditions, and novel ligand preparations to affect oligomerization. We have established 
that these unusual "monomers" display early alkyne-like metathesis behavior before forming novel polycyclic organometallic complexes.

Work has also been directed toward assessing the bulk response of organic materials to chemical and thermal stimuli for potential sensor and/or dynamic encapsulation applications. We have pursued the response of polyacetals to high and low $\mathrm{pH}$ environments under chemical amplification conditions, and established a new class of hybrid organic-inorganic materials containing thermally labile, Diels-Alder-reactive organic functionalities; the morphological character of these hybrids changes as a function of temperature.

\section{Results}

Results from this project were fruitful in assessing the utility of certain polymer systems for their autonomous response to optical, thermal and chemical stimuli. The participants on the project have made significant theoretical and experimental contributions in a variety of areas. These include preparing new materials systems and gaining insight into the predicted behavior of porphyrin-terminated polyenes by molecular modeling studies. Furthermore, limitations were established as to the utility of catalytic amounts of photogenerated acids and bases for the solid state bulk depolymerization of organic films and monoliths. Also, a likely mechanism to explain the behavior of phosphaalkynes in the presence of transition metal metathesis catalysts was established. The remainder of the report is a summary describing the research areas impacted by this LDRD: "Molecular Modeling of Polyene-Bridged Porphyrins," "Determination of $t$-BuC $\equiv \mathrm{P}$ Metathesis Activity with High Oxidation State Metal Alkylidenes," "Polyacetal Response to Photogenerated Acids and Bases" and "Thermally Responsive Materials."

\section{Molecular Modeling of Polyene-Bridged Porphyrins}

The molecular modeling component of the project has initially focused on potential organic conductors whose properties can be manipulated by their redox characteristics. Modeling these systems, as opposed to the thermal properties of diazo-containing sites, will allow us to investigate intelligent photo-, thermal- and redox controlled manipulation of organic systems.

One type of polymer of interest combines a conducting polymer with a photoactive redox center. We employed molecular simulation to design such a light-responsive 
polymer using photoactive metal porphyrins linked by polyacetylene. Polyacetylene serves as an initial model for computer simulations of other more interesting polymers. All-carbon conjugated systems with an increased degree of two-dimensional structure should offer the best prospect of maintaining interesting electrical and optical properties. As a first step in the computer-design of light-responsive polymers, we investigated the molecular and electronic structure of metal porphyrin linked by polyacetylene segments of varying length. Our initial calculations were designed to determine the spatial extent of the electronic interaction between two porphyrin chromophores through a conjugated polymer linker. Toward this end, we have computationally examined the conformational properties of a series of polymers in which two nickel(II)-porphine molecules are linked by polyacetylene molecules of differing lengths and conformations. These structures, shown schematically

\section{Figure 1}

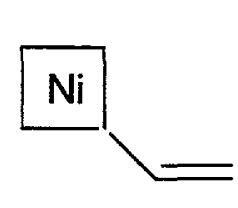

1

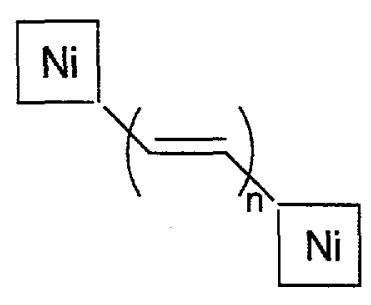

2; $n=1$

$4 ; n=3$
3; $n=2$

$5 ; n=4$

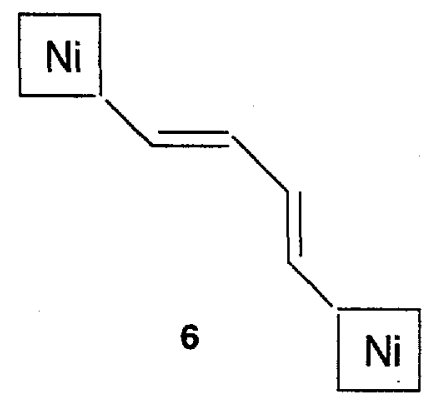

in Figure 1 with the Ni porphine represented by the squares, will be used as starting structures in INDO/s quantum-chemical calculations. We showed that nickel porphine is a planar molecule. However, we find that attaching a vinyl group destabilized the porphyrin macrocycle enough to cause a significant out-of-plane deformation (ruffling angles) which are affected by the spatial proximity of the other Ni-porphine within the same molecule. For two or more bridging ethylene groups, the deformation of the porphyrins is nearly constant.

The conformations of the molecules 1-6 are not planar as implied by Figure 1. Figure 2 shows the conformations of several of these molecules. Several low energy conformations of each molecule are stable (local minima). For example, there are both trans (3) and cis (6) isomers that depend on the conformation of the polyacetylene linker. We find the trans isomer is about $2 \mathrm{kcal} / \mathrm{mol}$ lower in energy than the cis. 


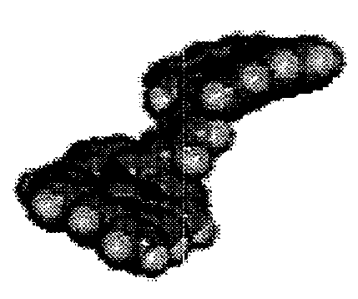

6

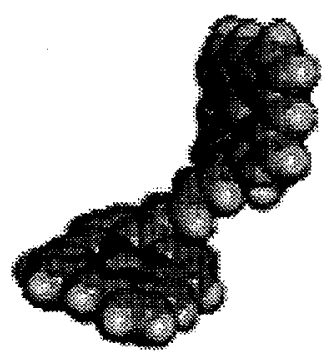

3-cis

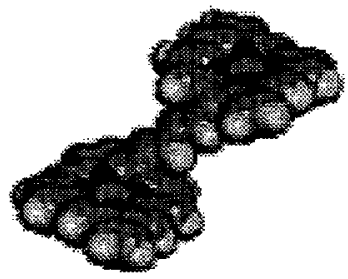

3-trans

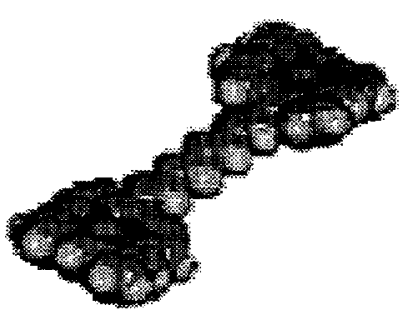

5

Figure 2. Low-energy stable conformers of polyacetylene linked porphyrins. The first three on the left have two intervening ethylene groups.

The stable conformers (local minima) of analogs of NiP-(ethylene) $-\mathrm{NiP}$, with $x=$ $1,2,4$, and 9 and where $\mathrm{NiP}$ is nickel(II) porphine, were calculated using a custom developed molecular mechanics force field. From these initial results, INDO/S and PM3 calculations of $\mathrm{NiP}$, meso-vinyl-NiP, and NiP-(ethylene) $)_{x} \mathrm{NiP}$ have been completed. The calculations show evidence of considerable delocalization of the frontier orbitals onto the bridging ethylene groups linking the two porphyrins. This is as was hoped, since photoreduction of the porphyrin (when $\mathrm{Sn}(\mathrm{TV}) \mathrm{Cl}_{2}$ is incorporated instead of $\mathrm{Ni}$ (II) into the porphyrin) could promote electron transfer along the polyacetylene linker between the porphyrins.
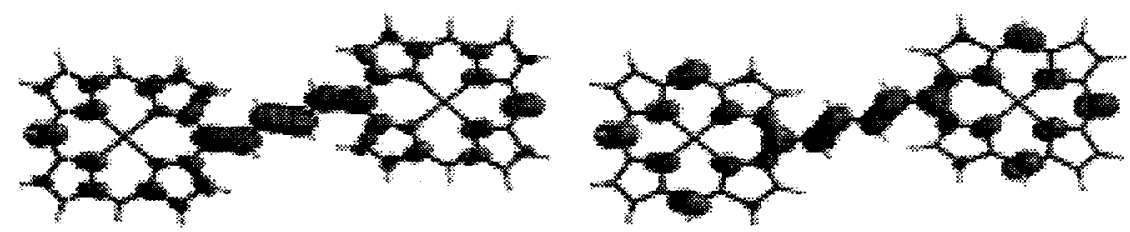

Figure 3. PM3-generated images of the LUMO (left) and HOMO (right) of the symmetric conformer of NiP-(ethylene) $)_{2}$-NiP showing delocalization of charge onto the ethylene groups linking the two porphyrins.

For $\mathrm{x}=1$, the top-filled (HOMO) is an $\mathrm{a}_{24}$-symmetry orbital and the lowest-empty (LUMO) is an $e_{z}$-symmetry orbital. Other frontier orbitals, including the other $a_{2 u}$ orbital, another component of the pair of $\mathrm{e}_{\mathrm{g}}$ orbitals, and one $\mathrm{a}_{1 \mathrm{u}}$ orbital, delocalize charge onto the ethylene linker. The charge is delocalized in the twisted conformer to about the same extent. Furthermore, charge delocalization is just as large when two ethylene groups are in the linker (Figure 3). 
The photophysical properties are influenced by extension of the conjugation of the system across the entire linked porphyrin dimer. For example, the predicted UV-visible absorption bands are red shifted more as the linker gets longer, as shown in Table 1. NiOEP-(ethylene) $)_{1}$-NiOEP (OEP = octaethylporphyrin) has a broad absorption that extends beyond $800 \mathrm{~nm}$, in agreement with the red-shifted absorption predicted by the calculations and a further red shift expected because of increased distortion for NiOEP compared with NiP. In contrast, the triplet (d,d) metal-centered state is almost constant for the linked porphyrins at about $6300 \mathrm{~cm}^{-1}$ above the ground state.

Table 1. Electronic transitions wavelengths ( $\mathrm{nm}$ ) and oscillator strengths of the UVvisible absorption bands of the porphyrin ring and the low-lying (d,d) state of the nickel ion from INDO/S calculations.

\begin{tabular}{|c|c|c|c|c|c|}
\hline \multirow[t]{3}{*}{ Compound } & \multicolumn{5}{|c|}{ Transition, nm (Oscillator Strength) } \\
\hline & \multicolumn{2}{|c|}{$\mathrm{Q}$} & \multicolumn{2}{|c|}{$\overline{\mathrm{B}}$} & \multirow[t]{2}{*}{$(\mathrm{d}, \mathrm{d})$} \\
\hline & $a_{2 u}$ & $a_{2 u}{ }^{\prime}$ & $a_{2 u}$ & $a_{2 u}^{\prime}$ & \\
\hline Meso-vinyl-NiP & $578(0.1)$ & $575(0.1)$ & $325(2.3)$ & $324(2.8)$ & 2024 \\
\hline $\mathrm{NiP}$-(ethylene) $)_{1} \mathrm{NiP}$ & $656(0.3)$ & $638(0.1)$ & & & 1583 \\
\hline $\mathrm{NiP}$-(ethylene) $)_{2}-\mathrm{NiP}_{\text {sym }}$ & $667(0.8)$ & $641(0.1)$ & & & 1590 \\
\hline $\mathrm{NiP}$-(ethylene) $)_{2}-\mathrm{NiP}_{\text {rwist }}$ & $659(0.4)$ & $636(0.1)$ & & & 1604 \\
\hline $\mathrm{NiP}$-(ethylene) $)_{9}-\mathrm{NiP}_{\text {sym }}$ & $767(5.0)$ & $640(0.1)$ & & & 1579 \\
\hline
\end{tabular}

We have synthesized several nickel and nickel-free analogs of the porphyrin dimers used in the calculation. These include (octaethylporphyrin) ${ }_{2}$ and (etioporphyrin) ${ }_{2}$ with either a single ethyl or ethylene linker between the two porphyrins. We also have synthesized the nickel derivative of (coproporphyrin-octa-ethylester) ${ }_{2}$ with an ethyl linker.

\section{Novel Reaction Pathways in the Reaction of $t-\mathrm{BuC} \equiv \mathrm{P}$ with High Oxidation State Metal Alkylidenes}

Electron deficient molybdenum alkylidenes react with 7 to directly form alkoxidemigrated head-to-tail $2+2$ cycloadducts 10a, $\mathbf{b}$ (Scheme 1 ); complexes ligated with bidentate alkoxide ligands also display reactivity to alkoxide-shifted products. Attenuation of electrophilicity (via altering the alkoxide substituent) permits the isolation of an intermediate molybdenum alkylidyne complex, $9 \mathrm{~b}$, which reflects cleavage of the phosphaalkyne $\mathrm{P} \equiv \mathrm{C}$ triple bond. Here we describe the extension of this approach to 
tungsten alkylidene $8 \mathrm{c}$ and the formation of a unique metallabicycle derived from the insertion of two equivalents of 7 into the $\mathrm{W}=\mathrm{C}$ tungsten alkylidene bond.

\section{Scheme 1 .}

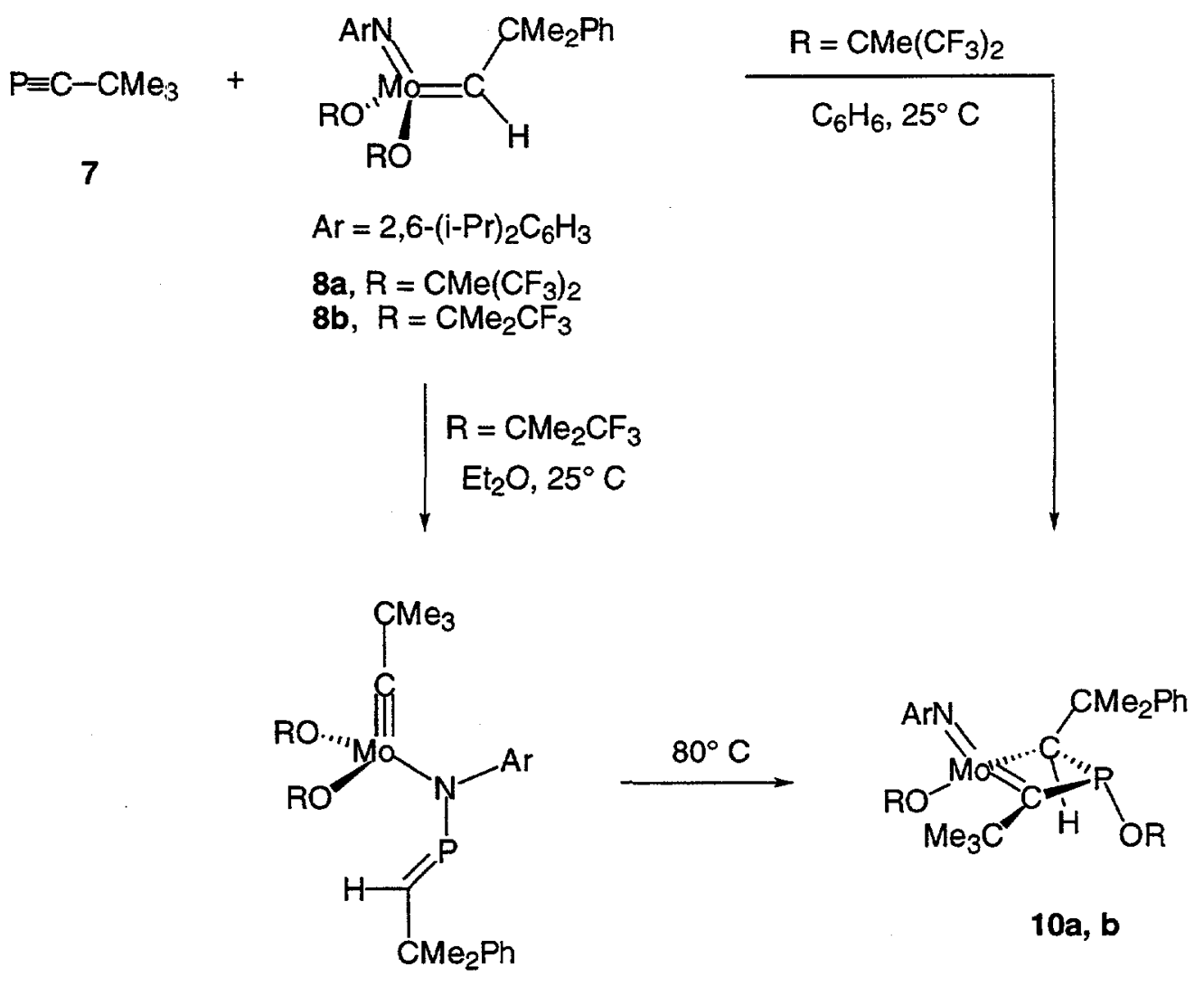

$9 b$

Introduction of 1.5 equivalents of $\mathrm{P} \equiv \mathrm{C}-t-\mathrm{Bu} 7$ to a bright yellow solution of tungsten alkylidene $8 \mathrm{c}$ at room temperature induces a color change to dark yellow and partial consumption of $\mathbf{8 c}$ (Scheme 2). Two new products are formed in an approximate 1:1 ratio. Complete consumption of starting alkylidene requires 3 equivalents of 7 . By ${ }^{1} \mathrm{H}$ NMR spectroscopy one set of product resonances was easily identified as the organotungsten analogue of the previously described family of molybdacyclobutenes, 10c. The second product was assisgned to complex 11. Isolation of, and spectral data from, $99 \%{ }^{13} \mathrm{C}$-labeled $11 *\left[99 \%{ }^{13} \mathrm{C}\right.$-enriched $t-\mathrm{Bu} * \mathrm{C} \equiv \mathrm{P}\left(7^{*}\right)$ was prepared from isotopically labeled ${ }^{*} \mathrm{CO}_{2}$ according to literature procedures] support a mechanism analogous to the Masuda alkylidene mechanism for polymerization of acetylenes, and unambiguously define the terminal olefinic group in 11* as being derived from the starting tungsten alkylidene complex. ${ }^{13} \mathrm{C}$ NMR of $11^{*}$ reflects the increased signal enhancement expected and allows 
assignment of the bridging $C_{\alpha}$ resonance (unobservable in natural abundance ${ }^{13} \mathrm{C}$ NMR spectrum).

We postulate a mechanism for formation of $10 \mathrm{c}$ and $\mathbf{1 1}$ which reflects a competing regiochemical preference of $\mathrm{P} \equiv \mathrm{C}-\mathrm{t}-\mathrm{Bu}$ for head-to-tail (leading to 10c) or head-to-head cycloaddition to the $\mathrm{W}=\mathrm{C}$ double bond (Scheme 3 ). Initial formation of head-to-tail cycloadduct 12, followed by 1,3-alkoxide migration to phosphorus, is an attractively simple explanation for generation of $10 \mathrm{c}$ and is consistent with ${ }^{13} \mathrm{C}$ NMR data obtained for 10c*. Also, this explanation has gained favor in arguments for products derived from phosphaalkyne metathesis by high oxidation state dinuclear tungsten complexes.

\section{Scheme 2}

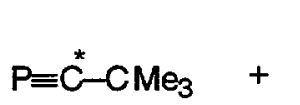

$7\left(7^{\star}\right)$

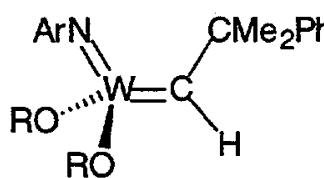

$\mathrm{Ar}=2,6-(\mathrm{i}-\mathrm{Pr})_{2} \mathrm{C}_{6} \mathrm{H}_{3}$

$8 \mathrm{c}, \mathrm{R}=\mathrm{CMe}\left(\mathrm{CF}_{3}\right)_{2}$

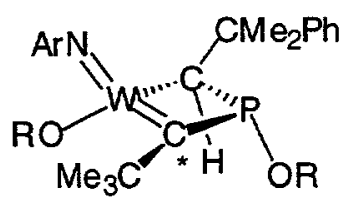

$10 \mathrm{c}$

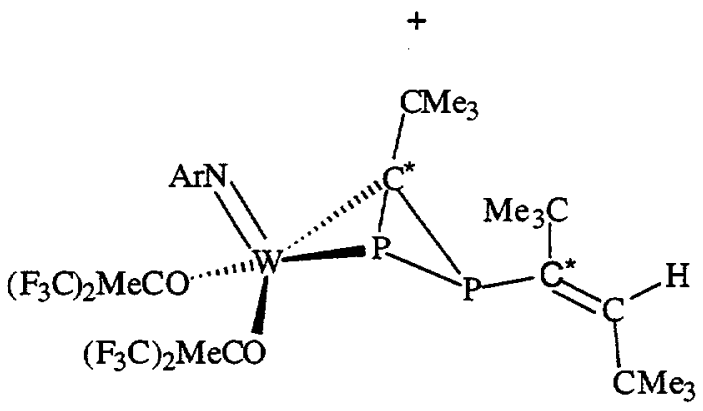

$11\left(11^{*}\right)$

Head-to-head cycloaddition of $\mathbf{7}$ to $\mathbf{8 c}$ followed by ring opening of the resultant phosphametallacyclobutene 13 would yield intermediate high oxidation state vinylphosphinidene complex 14 , which we anticipate should support $\mathrm{W}=\mathrm{P}$ multiple bonding and reflect reactivity as such. Cycloaddition of a second equivalent of 7 to phosphinidene 14, followed by a second ring opening, would yield a new phosphinidene intermediate; rearrangement of acyclic diphosphametallahexatriene 15 to observed bicyclic product 11 may be energetically favorable in light of the established reactivity of phosphaalkene $\mathrm{P}=\mathrm{C}$ double bonds and the relaxed ring strain energies inherent in $\mathbf{1 1}$ (vis- 


\section{Scheme 3}

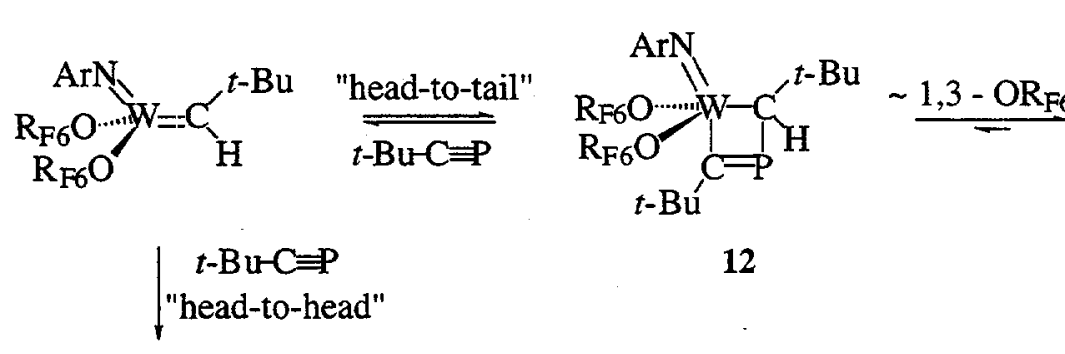

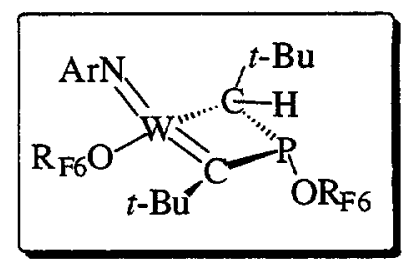

$10 c$

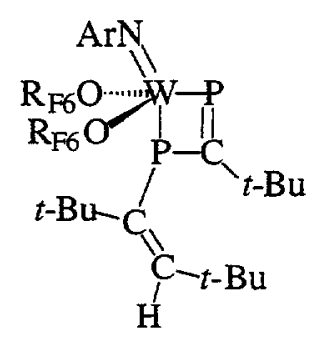

14
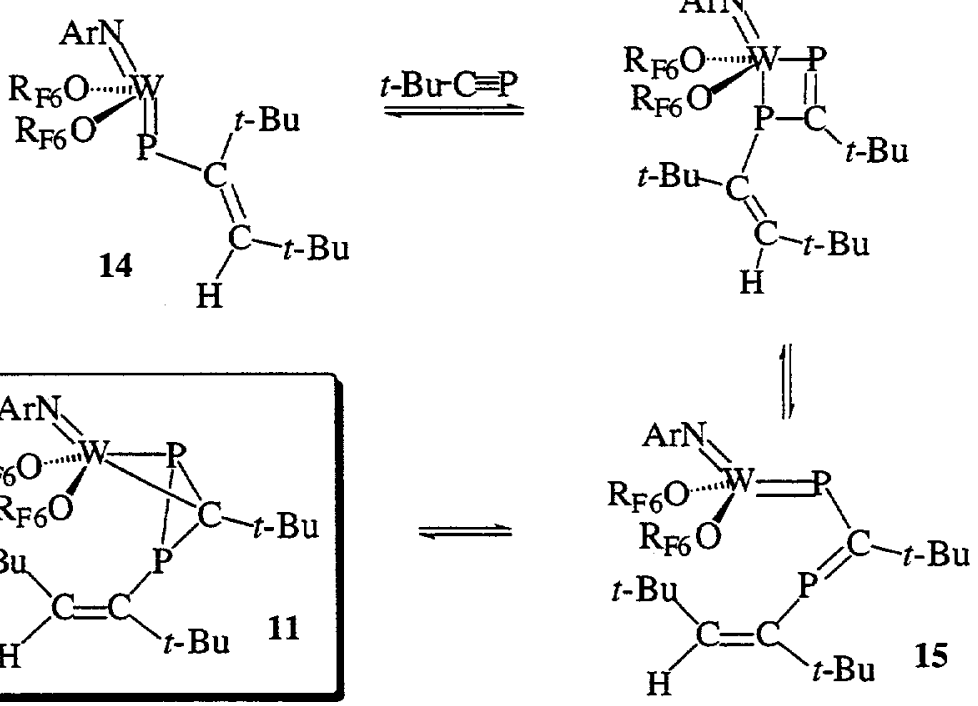

a-vis the organic bicyclobutane $\rightarrow$ butadiene system). Similar rearrangements have been invoked to explain the formation of diphosphabicyclobutanes from 2,3diphosphabutadienes.

\section{Polyacetal Response to Photogenerated Acids and Bases}

Chemical systems were all based on materials, such as the polyacetal of formaldehyde (below), with low ceiling temperatures (temperatures below which the equilibrium for the polymerization reaction favors monomeric species; Figure 4).

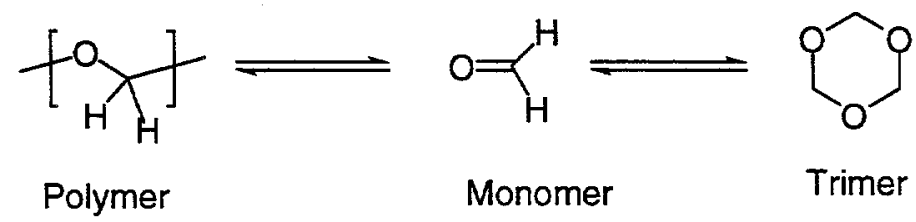

Figure 4. Equilibrium for polyformaldehyde with formaldehyde and trioxane. 
Examples of materials with their ceiling temperatures include polymethylstyrene (16, $\left.61^{\circ} \mathrm{C}\right)$, polyformaldehyde $\left(17,25^{\circ} \mathrm{C}\right)$, and polychloral $\left(18,58^{\circ} \mathrm{C}\right)$; see Figure 5 .

\section{Figure 5}

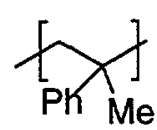

16

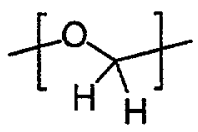

17

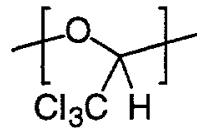

18

Polyformaldehyde or polyoxomethylene is a structural plastic formed from the Lewis acid or strong base-catalyzed polymerization of trioxane. We first examined the thermal degradation of polyformaldehyde to determine how similar the ceiling temperature was to the observed onset of degradation. These experiments were conducted using a thermal gravimetric analyzer. Polyformaldehyde depolymerized starting at $320^{\circ} \mathrm{C}, 300^{\circ} \mathrm{C}$ above its ceiling temperature, indicating that while the depolymerization may be thermodynamically favorable at room temperature, the activation barrier is sufficiently high to prevent facile reversion to the monomer.

Chemical amplification is an optical lithography strategy employed by the microprocessor industry for the delineation of well-defined sub-micron features in suitable polymeric photoresists. Catalytic amounts of photoacid generators, doped into polymeric matrices bearing acid-sensitive functionalities, act to alter the matrix polymer's solubility characteristics upon exposure to incident radiation, regenerating acid in the process. In this way, multiple equivalents of pendant acid-sensitive functional groups are altered from a small amount of photoactive dopant. The utility of employing polyacetals as $\mathrm{pH}$-sensitive matrix materials for coatings or encapsulants, which could be depolymerized in the bulk using photoacid- or photobase generators, was pursued.

We examined the effect of acidic catalysts on the depolymerization chemistry of polyacetals. Concentrated nitric acid did not lead to the catalytic depolymerization of the acetyl end-capped polyformaldehyde 17 . Instead, it would only react with the polymer stoichiometrically at temperatures $\left(75^{\circ} \mathrm{C}\right)$ well above the ceiling temperature. Nitrogen dioxide and water did not cause any depolymerization.

Polychloral or polytrichloroacetaldehyde (18) is another polyacetal with a relatively low ceiling temperature. This polymer is stable to acid catalysts, but is reportedly sensitive to bases. Thermal depolymerization would lead to the conversion of the insoluble polymer into the liquid chloral monomer. We showed that the polymer is exothermically reactive with organic bases such as n-butylamine, but that the reaction is stoichiometric, rather than 
catalytic. We also evaluated a variety of photo-amine generators (a family of orthonitrocarbamate esters) and thermal amine generators, such as ammonium carbonate, as catalysts for depolymerizing polychloral. No amine bases appear to be effective in depolymerizing the polychloral catalytically.

We synthesized a photo-hydroxide base generator by methylating nifedipine with methyl iodide (Scheme 4). N-Methyl nifedipine generates a pyridinium hydroxide upon<smiles>CCOC(=O)C1=C(C(=O)OC)NC(c2ccccc2[N+](=O)[O-])=C(C(C)=O)C1c1ccccc1[N+](=O)[O-]</smiles>

Scheme 4. Nifedipine is methylated by deprotonating with potassium hydride and quenching with methyl iodide.

exposure to ultraviolet light. The hydroxide base should be significantly stronger and more effective than amines in catalyzing the depolymerization of polychloral, but no such reaction was observed when mixtures of the $\mathrm{N}$-methyl nifedipine and polychloral were irradiated. In both cases, the polyacetals were completely immiscible with the catalysts. It appears likely that the heterogeneous nature of these systems is providing an effective kinetic barrier to depolymerization. In summary, a low ceiling temperature is clearly not the most critical factor in selecting a polymer as a thermally and or chemically responsive material.

\section{Thermally Responsive Materials}

We chose to investigate materials that would depolymerize thermally due to a relatively low ceiling temperature, but ones with a much lower activation barrier to depolymerization than the polyacetal systems, making the thermal response more of a thermodynamic issue rather than one controlled predominantly by the system's kinetics.

For this study we chose materials that were built using Diels-Alder chemistry. In this reaction, a monomer with two or more dienophilic groups, such as a maleimide, is reacted with a monomer with two or more dienyl groups in a Diels-Alder reaction to afford a polymer or polymeric network. The properties of the resulting polymer can be engineered through the selection of the appropriate functionalities incorporated into the 
monomeric building blocks. The reaction requires no initiator or additional reagents (or solvents) and can be viewed as a step-growth polymerization system (Scheme 5).<smiles>CC#CC</smiles>

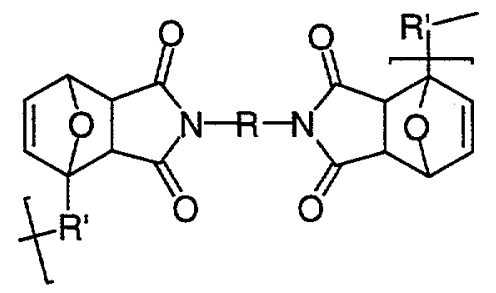

Scheme 5. Diels-Alder polymerization of a bis-maleimide functionalized monomer with a bis-furanyl-functionalized monomer.

One of the advantages of this system is that it converts a readily processible soluble, or perhaps liquid, precursor into a material that behaves as a thermoset or an elastomer, depending on what monomers are used. At lower temperatures the equilibrium of the Diels-Alder reaction lies towards the polymeric product. This means that the polymerization proceeds as expected at ambient or slightly elevated temperature. At higher temperatures the equilibrium shifts back to the left and the polymer depolymerizes to regenerate soluble or liquid monomers. The end result is a class of materials that acts as a thermoplastic-thermoset. The materials have many of the properties of thermosets such as epoxies after polymerization or curing. Unlike classical thermosets, however, these reversible polymers become processible again with heating (Scheme 6).

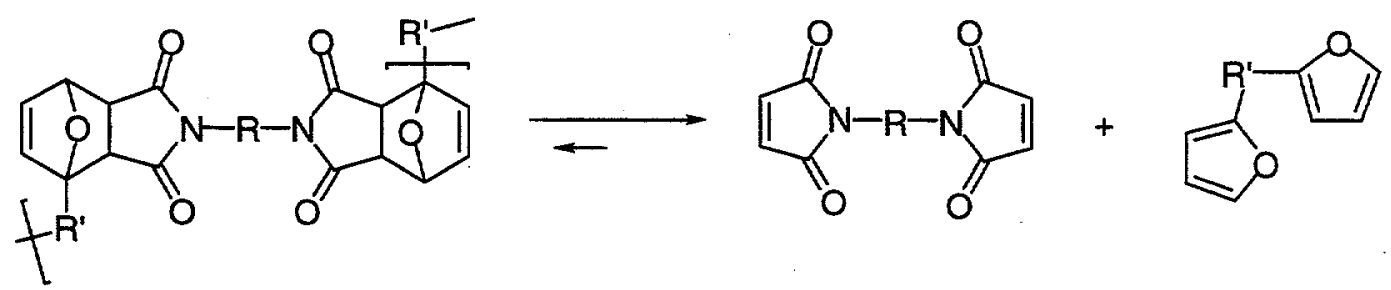

Scheme 6. Thermal depolymerization of the Diels-Alder polymer to afford small soluble or liquid products.

The focus of the work in this project was to: 1) prepare these thermally removable materials with a well-defined synthetic filler phase based on polysilsesquioxanes as part of the material; and 2) develop a new technique for rapidly building a library of systems with different depolymerization temperatures. 


\section{Maleimide-Functionalized Polysilsesquioxanes}

The first goal was achieved by preparing maleimide-functionalized alkoxysilane monomers that could be hydrolyzed and condensed at the silicon to give highly branched polysilsesquioxane "filler phases" covered with dienophilic groups capable of reacting with dienes to afford hybrid organic-inorganic materials (Scheme 7). Alternatively, we found that the maleimide groups could be thermally auto-polymerized, polymerized with a radical initiator and co-polymerized with vinyl monomers such as styrene to give different types of hybrid organic-inorganic materials. The maleimide-functionalized polysilsesquioxanes could be prepared with a high degree of control over the molecular weight, allowing the size of the filler domains to be pre-determined.

The resulting hybrid materials could readily be prepared as transparent monoliths. One drawback to the maleimide-functionalized system is the reactivity of the maleimide group to nucleophilic or Michael additions that would render the groups useless for DielsAlder or radical chemistry. The aqueous conditions used to polymerize the alkoxysilane group were not suitable for retention of the stand-alone maleimide functionality. However, we found that by "protecting" the maleimide as the Diels-Alder adduct with furan, the solgel polymerization to form the polysilsesquioxane could proceed without incident (Scheme 8). Heating this oxonorbornene-functionalized polysilsesquioxane initiates the retro-Diels-

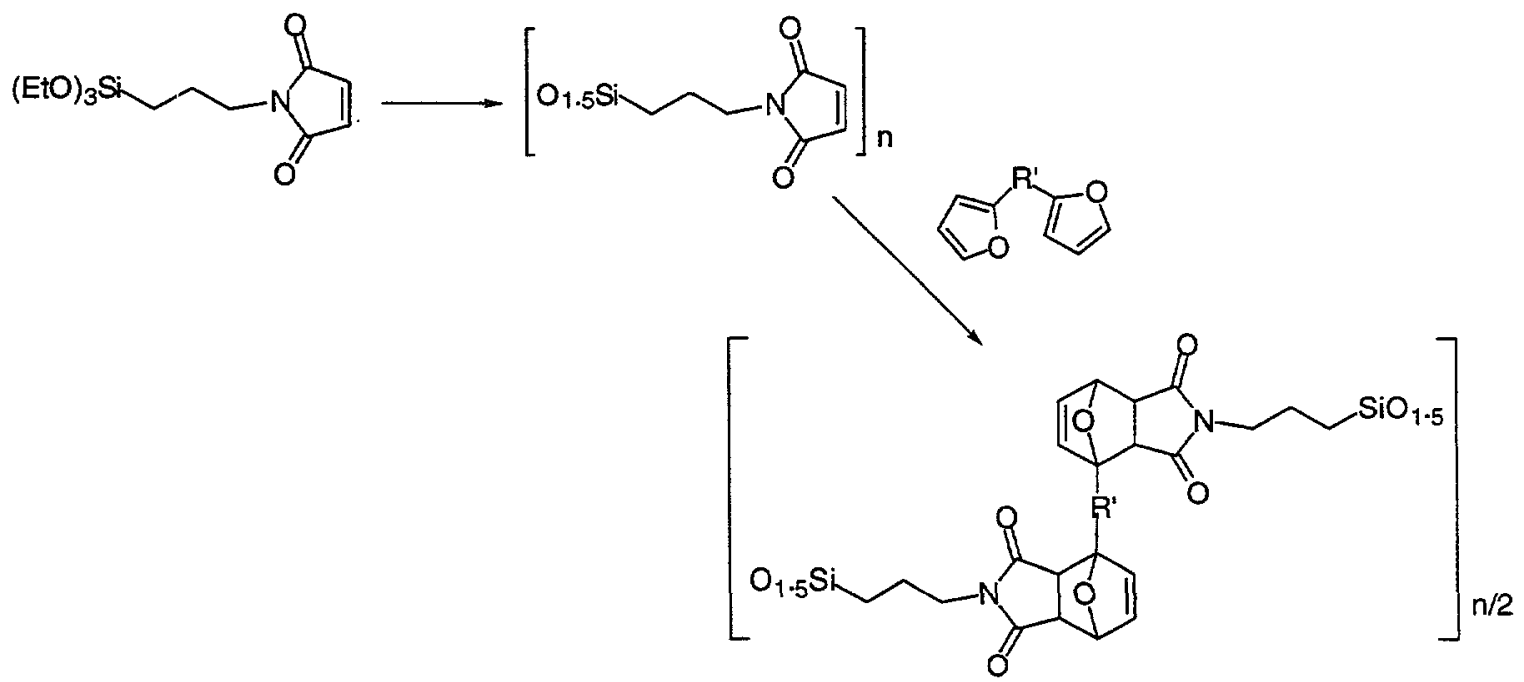

Scheme 7. Preparation and Diels-Alder Polymerization of maleimide-functionalized polysilsesquioxanes. 


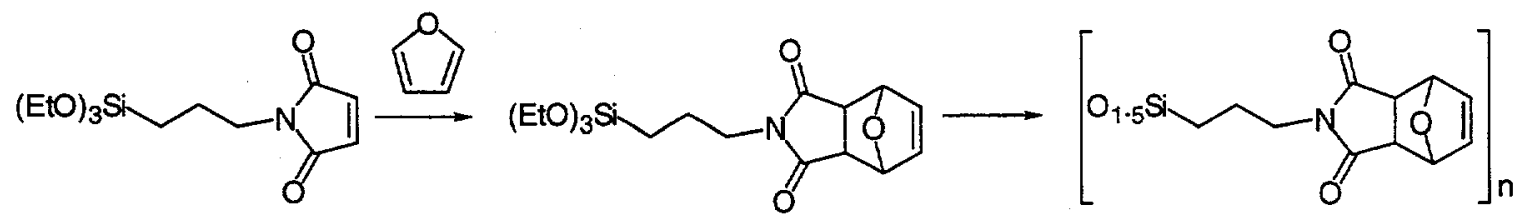

Scheme 8. Preparation of a "protected" maleimide functionalized polysilsesquioxane.

Alder reaction to re-generate the maleimide which could then undergo Diels-Adler chemistry with alternative diene co-monomers or other free radical homo- or copolymerization reactions (Scheme 9).

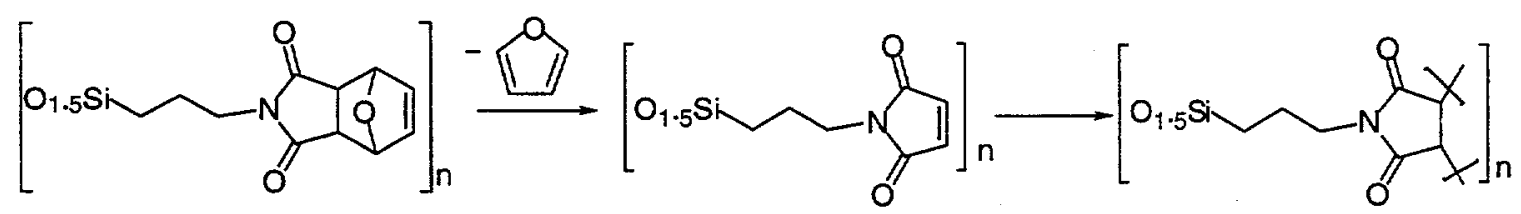

Scheme 9. Generalized deprotection and free radical auto-polymerization of the maleimide groups.

Polymers with Diels-Alder linkages were found to thermally "depolymerize" to give the precursor polysilsesquioxanes at around $90^{\circ} \mathrm{C}$. Those materials prepared by the autopolymerization of the maleimide or its copolymerization with vinyl monomers were stable to over $300^{\circ} \mathrm{C}$.

\section{Rapid Discovery of Diels-Alder Linkages}

The maleimide-furan Diels-Alder polymers described above depolymerize at around $90-110^{\circ} \mathrm{C}$. It would be advantageous to have similar reversible systems with higher depolymerization temperatures for applications with higher service temperatures. The temperature at which these depolymerizations occur will be the product of the thermodynamics and the kinetics of the reaction. It is known that the temperature at which the forward Diels-Alder reaction proceeds is dependent on the substituents attached to the dienophile and diene precursors. However, there are few published reports detailing how those substituent effects influence the temperature at which retro Diels-Alder depolymerization reactions will occur. One method to determine this substituent effect would be to prepare a family of polymers, each based on different Diels-Alder adducts, and experimentally determine the depolymerization temperatures by differential scanning calorimetry or differential mechanical analysis. However, this would be a time consuming process. 
In the last year of this LDRD we began to develop a combinatorial method for quickly screening large numbers of Diels-Alder linkages and determining the temperatures at which those linkages break apart. The technique uses a polymer with a single type of dienophilic substituent as a pendant functionality suspended along the length of the polymeric chain. This polymer would be reacted with many different monomeric dienes all in the same reaction pot (Scheme 10). The resulting polymer, now functionalized with a family of different Diels-Alder adducts, would be purified and then analyzed by thermal gravimetric analysis using a very slow temperature ramp in conjunction with a mass spectrometric analyzer. The TGA-MS would allow the exact temperature ranges for the different retro Diels-Alder reactions to be determined simply by measuring the temperature range at which the different dienophiles were released. In the same fashion a polymer bearing a single type of diene could be reacted with many dienophiles in a single experiment (Scheme 11).

Once the table of retro-Diels-Alder temperatures for the different diene-dienophile combinations has been filled out, polymers based on the Diels-Alder linkages can be prepared and the retro Diels-Alder depolymerization temperatures measured to validate the combinatorial technique. We have prepared both dienophile and diene functionalized polymers and have reacted the polymers with families of dienes and dienophiles, respectively. We are presently waiting to perform the thermal analysis experiments.

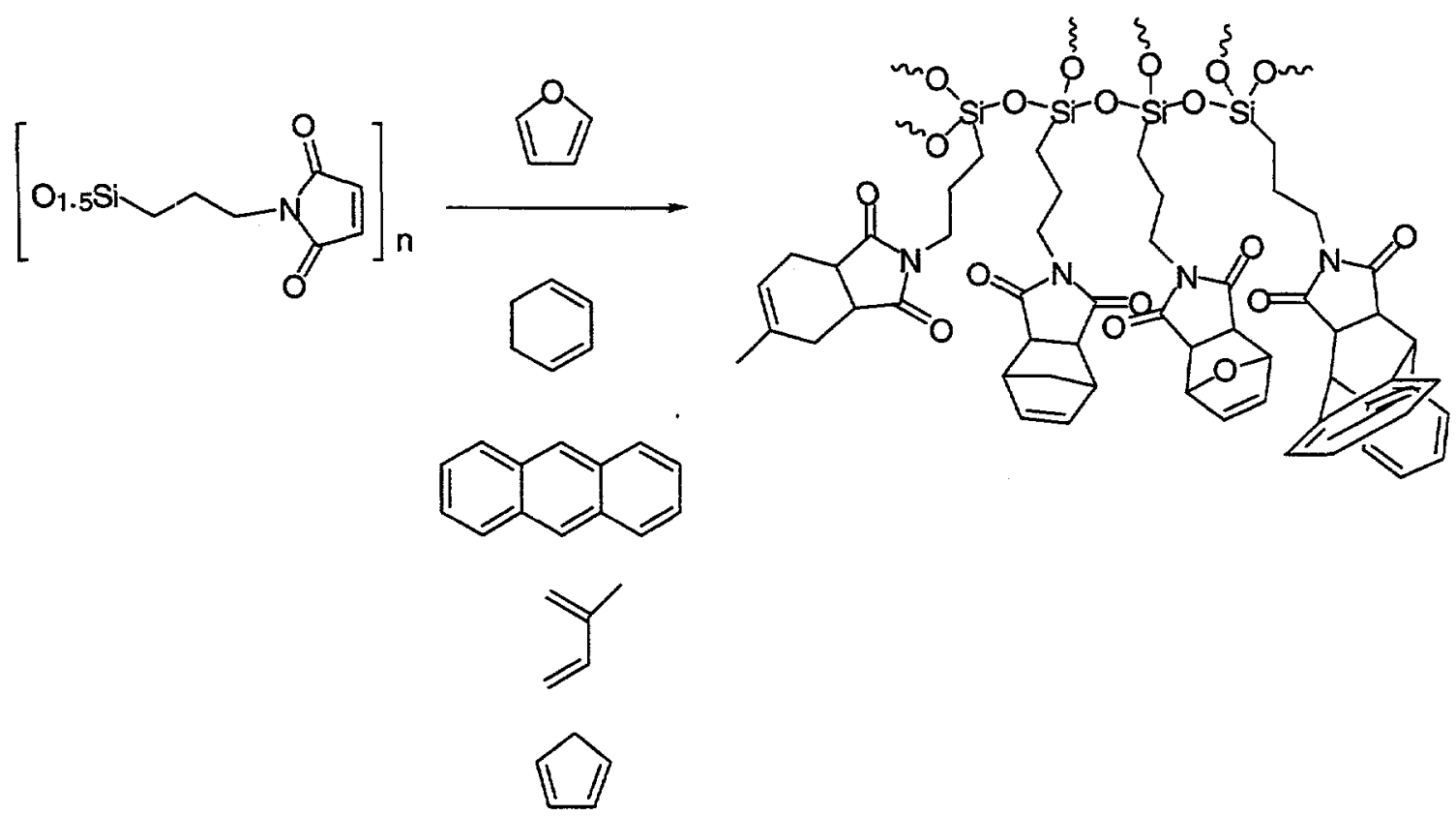

Scheme 10. Dienophile-functionalized polymer reacting with number of dienes to give polymer possessing a combinatorial library of pendant Diels-Alder adducts. 


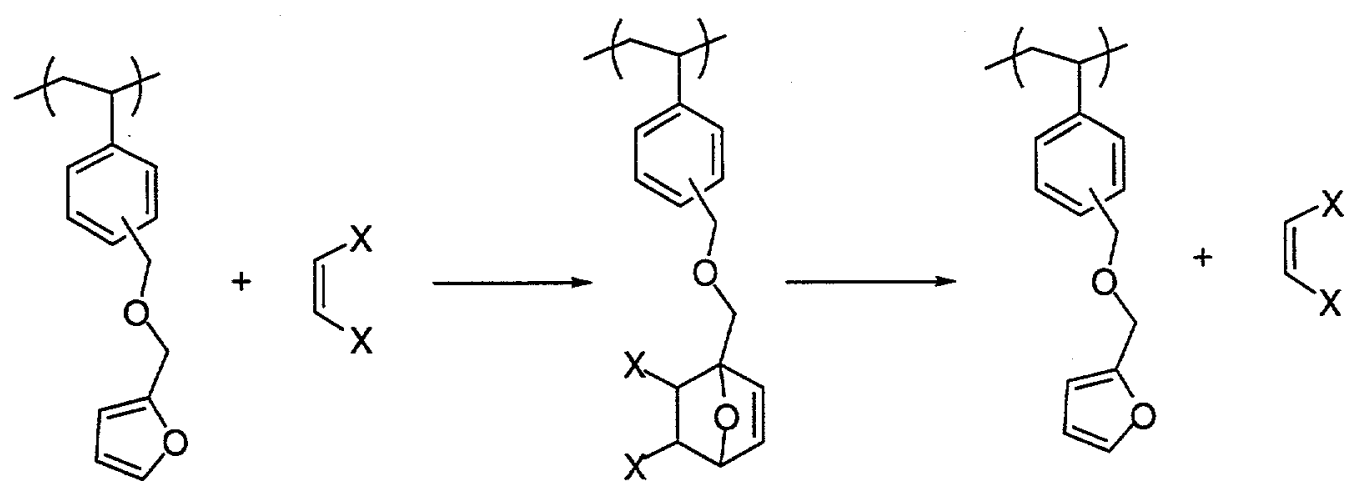

Scheme 11. Diene-functionalized polymer reacting with number of dienophiles to give polymer possessing a complementary combinatorial library of pendant Diels-Alder adducts.

\section{Summary}

The research conducted as part of this LDRD project has made significant contributions in several areas of science. Molecular models of polyene-bridged metal porphyrin complexes predict that conductivity through the polyene $\pi$-system is possible, as there should be delocalization of charge carrier density from the porphyrin macrocycle to the unsaturated polyene moiety. Detailed investigations of phosphaalkyne reactivity in the presence of transition metal metathesis catalysts support the theory that phosphaalkynes oligomerize similarly to polyacetylene precursors. New organometallic complexes bearing cyclic bidentate alkoxide ligands have not prevented alkoxide shift product formation to date. Polyacetal polymers do not show depolymerization behavior to catalytic amounts of acid or base in the solid state, presumably due to system heterogeneity and high activation barriers to depolymerization. However, Diels-Alder functionalized hybrid materials have been prepared which display thermoreversibility at the diene/dienophile moiety; the potential for exploring large libraries of new hybrids for specific thermal characteristics has been established via preliminary combinatorial experiments.

\section{Acknowledgments}

The authors would like to thank Martin Peterson and Duane Schneider for monomer and polymer syntheses and spectral characterizations. This work was supported by the United States Department of Energy under contract DE-AC04-94AL85000. Sandia is a multiprogram laboratory operated by Sandia Corporation, a Lockheed Martin Company, for the United States Department of Energy. 


\section{UNLIMITED RELEASE}

\section{INITIAL DISTRIBUTION}

\section{Copies}

MS 1206

1

M. J. Carr

MS 1349

1

J. A. Shelnutt

MS 1407

1

G. M. Jamison

MS 1407

1

MS 1407

1

D. A. Loy

MS 1407

1

D. R. Wheeler

MS 1407

1

J. Aubert

MS 1435

1

MS 0161

1

MS 0188

MS 0612

MS 0899

MS 9018

R. L. Clough

A. K. Hays

Patent and Licensing Office, Org. 4331

LDRD Office, Org. 4001

Review and Approval Desk, Org. 4912

For DOE/OSTI

Technical Library, Org. 4916

Central Technical Files, Org. 8940-2 